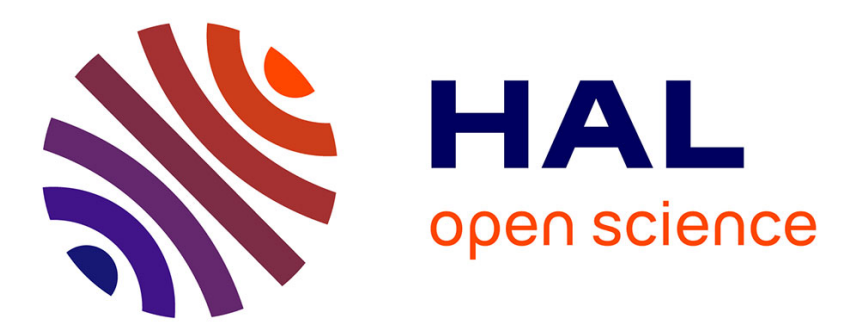

\title{
3D COLORED MESH GRAPH SIGNALS MULTI-LAYER MORPHOLOGICAL ENHANCEMENT
}

Olivier Lézoray

\section{- To cite this version:}

Olivier Lézoray. 3D COLORED MESH GRAPH SIGNALS MULTI-LAYER MORPHOLOGICAL ENHANCEMENT. 42nd IEEE International Conference on Acoustics, Speech and Signal Processing (ICASS P2017), May 2017, New Orleans, LA, United States. hal-01486190

\author{
HAL Id: hal-01486190 \\ https://hal.science/hal-01486190
}

Submitted on 9 Mar 2017

HAL is a multi-disciplinary open access archive for the deposit and dissemination of scientific research documents, whether they are published or not. The documents may come from teaching and research institutions in France or abroad, or from public or private research centers.
L'archive ouverte pluridisciplinaire HAL, est destinée au dépôt et à la diffusion de documents scientifiques de niveau recherche, publiés ou non, émanant des établissements d'enseignement et de recherche français ou étrangers, des laboratoires publics ou privés. 


\title{
3D COLORED MESH GRAPH SIGNALS MULTI-LAYER MORPHOLOGICAL ENHANCEMENT
}

\author{
Olivier Lézoray \\ Normandie Univ, UNICAEN, ENSICAEN, CNRS, GREYC, 14000 Caen, France
}

\begin{abstract}
We address the problem of sharpness enhancement of 3D colored meshes. The problem is modeled with graph signals and their morphological processing is considered. A hierarchical framework that decomposes the graph signal into several layers is introduced. It relies on morphological filtering of graph signal residuals at several scales. To have an efficient sharpness enhancement, the obtained layers are blended together with the use of a nonlinear sigmoid detail enhancement and tone manipulation, and of a structure mask.
\end{abstract}

Index Terms - Graph signal, morphology, color, multilayer decomposition, detail enhancement, sharpness.

\section{INTRODUCTION}

3D Meshes are widely used in many fields and applications such as computer graphics and games. Recently, low cost sensors have brought 3D scanning into the hands of consumers. As a consequence, a new market has emerged that proposes cheap software that, similarly to an ordinary video camera, enables to generate 3D models by simply moving around an object or a person. With such software one can now easily produce $3 \mathrm{D}$ colored meshes with each vertex described by its position and color. However, the quality of the mesh is not always visually good. In such a situation, the sharpness of the 3D colored mesh needs to be enhanced. In this paper we propose an approach towards this problem. Existing techniques for sharpness enhancement of images use structurepreserving smoothing filters $[1,2,3,4]$ within a hierarchical framework. They decompose the image into different layers from coarse to fine details, making it easier for subsequent detail enhancement. Some filters have been extended to 3D meshes but most manipule only mesh vertices positions $[5,6]$. Some recent works have considered the color information [7]. In this paper we present a robust sharpness enhancement technique based on morphological signal decomposition. The approach considers manifold-based morphological operators to construct a complete lattice of vectors. With this approach, a multi-layer decomposition of the 3D colored mesh, modeled

This work received funding from the Agence Nationale de la Recherche (ANR-14-CE27-0001 GRAPHSIP), and from the European Union FEDER/FSE 2014/2020 (GRAPHSIP project). as a graph signal, is proposed that progressively decomposes an input color mesh from coarse to fine scales. The layers are manipulated by non-linear s-curves and blended by a structure mask to produce an enhanced 3D color mesh. The paper is organized as follows. In Section 2, we introduce a learned ordering of the vectors of a graph signal. From this ordering, we derive a graph signal representation and define the associated morphological graph signal operators. Section 3 describes the proposed method for multi-layer morphological enhancement of graph signals. Last sections present results and conclusion.

\section{MATHEMATICAL MORPHOLOGY FOR 3D COLORED GRAPH SIGNALS}

\subsection{Notations}

A graph $\mathcal{G}=(\mathcal{V}, \mathcal{E})$ consists in a set $\mathcal{V}=\left\{v_{1}, \ldots, v_{m}\right\}$ of vertices and a set $\mathcal{E} \subset \mathcal{V} \times \mathcal{V}$ of edges connecting vertices. A graph signal is a function that associates real-valued vectors to vertices of the graph $f: \mathcal{G} \rightarrow \mathcal{T} \subset \mathbb{R}^{n}$ where $\mathcal{T}$ is a non-empty set of vectors. The set $\mathcal{T}=\left\{\mathbf{v}_{1}, \cdots, \mathbf{v}_{m}\right\}$ represents all the vectors associated to all vertices of the graph (we will also use the notation $\mathcal{T}[i]=\mathbf{v}_{i}=f\left(v_{i}\right)$ ). In this paper 3D colored graphs signals are considered, where a color is assigned to each vertex of a triangulated mesh.

\subsection{Manifold-based color ordering}

Morphological processing of graph signals requires the definition of a complete lattice $(\mathcal{T}, \leq)$ [8], an ordering of all the vectors of $\mathcal{T}$. Since there exits no admitted universal ordering fo vectors, the framework of $h$-orderings [9] has been proposed as an alternative. This consists in constructing a bijective projection $h: \mathcal{T} \rightarrow \mathcal{L}$ where $\mathcal{L}$ is a complete lattice equipped with the conditional total ordering [9]. We refer to $\leq_{h}$ as the $h$-ordering given by $\mathbf{v}_{i} \leq_{h} \mathbf{v}_{j} \Leftrightarrow h\left(\mathbf{v}_{i}\right) \leq h\left(\mathbf{v}_{j}\right)$. As argued in our previous works [10], the projection $h$ cannot be linear since a distortion of the space topology is inevitable. Therefore, it is preferable to rely on a nonlinear mapping $h$. The latter will be constructed by learning the manifold of vectors from a given graph signal and the complete lattice $\left(\mathcal{T}, \leq_{h}\right)$ will be deduced from it. 


\subsubsection{Complete lattice learning}

Given a graph signal that provides a set $\mathcal{T}$ of $m$ vectors in $\mathbb{R}^{3}$, a dictionary $\mathcal{D}=\left\{\mathbf{x}_{1}^{\prime}, \cdots, \mathbf{x}_{p}^{\prime}\right\}$ of $p \ll m$ vectors is built by Vector Quantization [11]. A similarity matrix $\mathbf{K}_{\mathcal{D}}$ that contains the pairwise similarities between all the dictionary vectors $\mathbf{x}_{i}^{\prime}$ is then computed. The manifold of the dictionary vectors is modeled using nonlinear manifold learning by Laplacian Eigenmaps [12]. This is be performed with the decomposition $\mathbf{L}=\boldsymbol{\Phi}_{\mathcal{D}} \boldsymbol{\Pi}_{\mathcal{D}} \boldsymbol{\Phi}_{\mathcal{D}}^{T}$ of the normalized Laplacian matrix $\mathbf{L}=\mathbf{I}-\mathbf{D}_{\mathcal{D}}^{-\frac{1}{2}} \mathbf{K}_{\mathcal{D}} \mathbf{D}_{\mathcal{D}}^{-\frac{1}{2}}$ with $\boldsymbol{\Phi}_{\mathcal{D}}$ and $\boldsymbol{\Pi}_{\mathcal{D}}$ its eigenvectors and eigenvalues, and $\mathbf{D}_{\mathcal{D}}$ the degree diagonal matrix of $\mathbf{K}_{\mathcal{D}}$. The obtained representation being only valid for the dictionary $\mathcal{D}$, it is extrapolated to all the vectors of $\mathcal{T}$ by Nyström extrapolation [13] expressed by $\tilde{\boldsymbol{\Phi}}=\mathbf{D}_{\mathcal{D} \mathcal{T}}^{-\frac{1}{2}} \mathbf{K}_{\mathcal{D} \mathcal{T}}^{T} \mathbf{D}_{\mathcal{D}}^{-\frac{1}{2}} \boldsymbol{\Phi}_{\mathcal{D}}(\operatorname{diag}[\mathbb{1}]-$ $\left.\boldsymbol{\Pi}_{\mathcal{D}}\right)^{-1}$, where $\mathbf{K}_{\mathcal{D} \mathcal{T}}$ is the similarity matrix between sets $\mathcal{D}$ and $\mathcal{T}$, and $\mathbf{D}_{\mathcal{D} \mathcal{T}}$ its associated diagonal degree matrix. Finally, the bijective projection $h: \mathcal{T} \subset \mathbb{R}^{3} \rightarrow \mathcal{L} \subset \mathbb{R}^{p}$ on the manifold is defined as $h(\mathbf{x})=\left(\tilde{\phi}^{1}(\mathbf{x}), \cdots, \tilde{\phi}^{p}(\mathbf{x})\right)^{T}$ with $\tilde{\phi}^{k}$ the $k$-th eigenvector. The complete lattice $\left(\mathcal{T}, \leq_{h}\right)$ is obtained by using the conditional ordering after this projection.

\subsubsection{Graph signal representation}

The complete lattice $\left(\mathcal{T}, \leq_{h}\right)$ being learned, a new graph signal representation can be defined. Let $\mathcal{P}$ be a sorted permutation of the elements of $\mathcal{T}$ according to the manifold-based ordering $\leq_{h}$, one has $\mathcal{P}=\left\{\mathbf{v}_{1}^{\prime}, \cdots, \mathbf{v}_{m}^{\prime}\right\}$ with $\mathbf{v}_{i}^{\prime} \leq_{h} \mathbf{v}_{i+1}^{\prime}$, $\forall i \in[1,(m-1)]$. From this ordered set of vectors, an index graph signal can be defined. Let $I: \mathcal{G} \rightarrow[1, m]$ denote this index graph signal. Its elements are defined as $I\left(v_{i}\right)=\left\{k \mid \mathbf{v}_{k}^{\prime}=f\left(v_{i}\right)=\mathbf{v}_{i}\right\}$. Therefore, at each vertex $v_{i}$ of the index graph signal $I$, one obtains the rank of the original vector $f\left(v_{i}\right)$ in $\mathcal{P}$, the set of sorted vectors, that we will call a palette. A new representation of the original graph signal $f$ is obtained and denoted in the form of the pair $f=(I, \mathcal{P})$. Figure 1 presents such a representation for a $3 \mathrm{D}$ colored graph signal. The original graph signal $f$ can be directly recovered since $f\left(v_{i}\right)=\mathcal{P}\left[I\left(v_{i}\right)\right]=\mathcal{T}[i]=\mathbf{v}_{i}$.

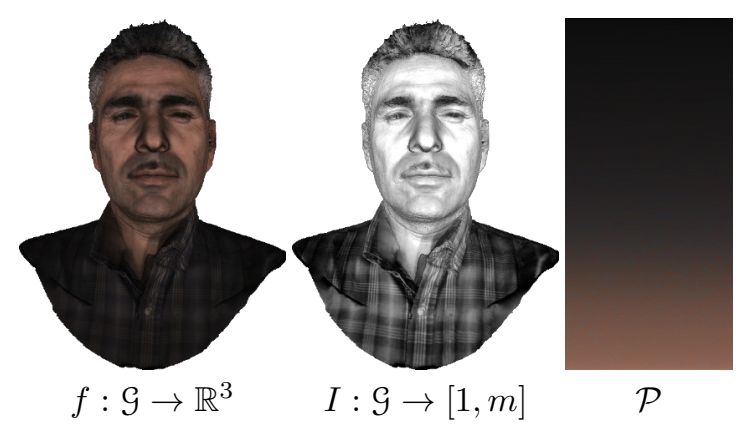

Fig. 1. From left to right: a $3 \mathrm{D}$ colored graph signal $f$, and its representation in the form of an index graph signal $I$ and associated sorted vectors $\mathcal{P}$.

\subsection{Graph signal morphological processing}

From this new representation of graph signals, morphological operators can now be expressed for the latter. The erosion of a graph signal $f$ at vertex $v_{i} \in \mathcal{G}$ by a structuring element $B_{k} \subset \mathcal{G}$ is defined as: $\epsilon_{B_{k}}(f)\left(v_{i}\right)=\left\{\mathcal{P}\left[\wedge I\left(v_{j}\right)\right], v_{j} \in\right.$ $\left.B_{k}\left(v_{i}\right)\right\}$. The dilation $\delta_{B_{k}}(f)\left(v_{i}\right)$ can be defined similarly. A structuring element $B_{k}\left(v_{i}\right)$ of size $k$ defined at a vertex $v_{i}$ corresponds to the $k$-hop set of vertices that can be reached from $v_{i}$ in $k$ walks, plus vertex $v_{i}$. These graph signal morphological operators operate on the index graph signal $I$, and the processed graph signal is reconstructed through the sorted vectors $\mathcal{P}$ of the learned complete lattice. From these basic operators, we can obtain other morphological filters for graph signals such a as openings $\gamma_{B_{k}}(f)=\delta_{B_{k}}\left(\epsilon_{B_{k}}(f)\right)$ and closings $\phi_{B_{k}}(f)=\epsilon_{B_{k}}\left(\delta_{B_{k}}(f)\right)$.

\section{MULTI-LAYER MORPHOLOGICAL ENHANCEMENT}

\subsection{Graph signal multi-layer decomposition}

We adopt the strategy of [14] that consists in decomposing a signal into a base layer and several detail layers, each capturing a given scale of details. We propose the following multiscale morphological decomposition of a graph signal into $l$ layers, as shown in Algorithm 1. To extract the successive

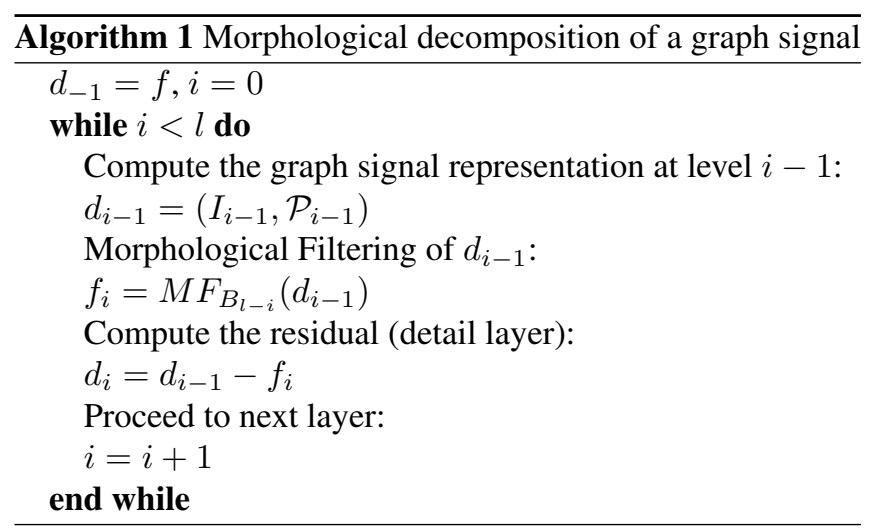

layers in a coherent manner, the layer $f_{0}$ has to be the coarsest version of the graph signal, while the residuals $d_{i}$ have to contain details that become finer across the decomposition levels. This means that the sequence of scales should be decreasing and therefore the size of the structuring element in the used morphological filtering (MF) should also decrease. In terms of graph signal decomposition, this means that as the process evolves, the successive decompositions extract more details from the original graph signal (similarly as [15]). In Algorithm 1, this is expressed by $B_{l-i}$ which is a sequence of structuring elements of decreasing sizes with $i \in[0, l-1]$. Since each detail layer $d_{i}$ is composed of a set of vectors different from the previous layer $d_{i-1}$, the graph signal representation $\left(I_{i}, \mathcal{P}_{i}\right)$ has to be computed for the successive lay- 


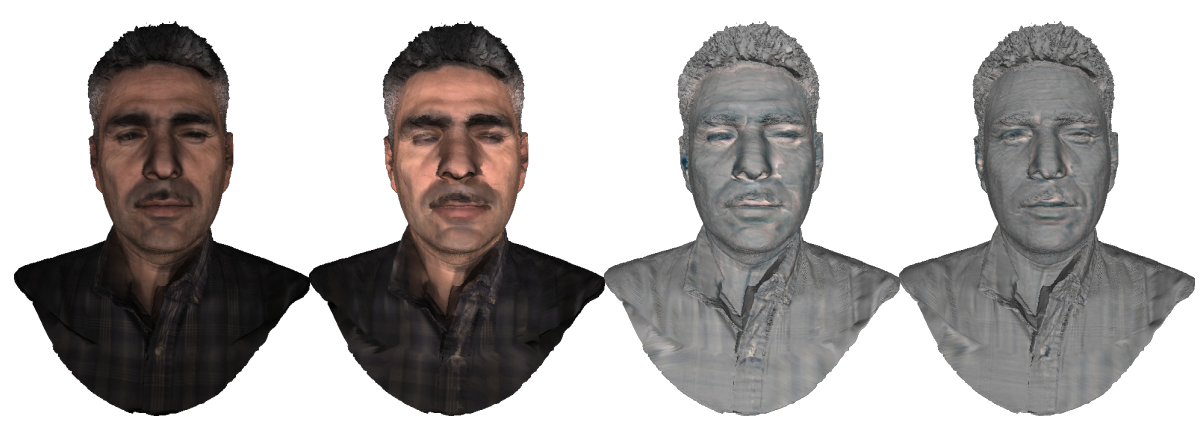

Fig. 2. From top to bottom, left to right: an original mesh $f$, and its decomposition into three layers $f_{0}, f_{1}$, and $d_{1}$.

ers to decompose. Finally, the graph signal can then be represented by $f=\sum_{i=0}^{l-2} f_{i}+d_{l-1}$. The $f_{i}$ 's thus represent different layers of $f$ captured at different scales. The morphological filter we have considered for the decomposition is an Open Close Close Open. The OCCO filter is a self-dual operator that has excellent signal decomposition abilities [16]: $O C C O_{B_{k}}(f)=\frac{\gamma_{B_{k}}\left(\phi_{B_{k}}(f)\right)+\phi_{B_{k}}\left(\gamma_{B_{k}}(f)\right)}{2}$. In Figure 2, we show an example with three levels of decomposition $(l=3)$ to obtain a coarse base layer $f_{0}$, a medium detail layer $f_{1}$ and a fine detail layer $d_{1}$.

\subsection{Graph signal enhancement}

\subsubsection{Proposed approach}

Given a graph signal $f=(I, \mathcal{P})$, we first construct its multilayer decomposition in $l$ levels. The graph signal can be enhanced by manipulating the different layers with specific coefficients and adding the modified layers altogether. This is achieved with the following proposed scheme:

$$
\hat{f}\left(v_{k}\right)=S_{0}\left(f_{0}\left(v_{k}\right)\right)+M\left(v_{k}\right) \cdot \sum_{i=1}^{l-1} S_{i}\left(f_{i}\left(v_{k}\right)\right) .
$$

with $f_{l-1}=d_{l-1}$. Each layer is manipulated by a nonlinear function $S_{i}$ for detail enhancement and tone manipulation. The layers are combined with the use of a structure mask $M$ that prevents from boosting noise and artifacts while enhancing the main structures of the original graph signal $f$. We provide now details on $S_{i}$ and $M$.

\subsubsection{Nonlinear boosting curve}

In classical image detail manipulation, the layers are manipulated in a linear way with specific layer coefficients (i.e., $S_{i}(x)=\alpha_{i} x$ [17]). However this can over-enhance some image details and requires hard clipping. Therefore, alternative nonlinear detail manipulation and tone manipulation have ben proposed [14, 18, 19]. Similarly, we consider a nonlinear sigmoid function of the form $S_{i}(x)=\frac{1}{1+\exp \left(-\alpha_{i} x\right)}$, appropriately shifted and scaled. The parameter $\alpha_{i}$ of the sigmoid is automatically determined and decreases while $i$ increases, whereas its width increases from one level to the other (details not provided due to reduced space).

\subsubsection{Structure mask}

As recently proposed in [19] for image enhancement, it is much preferable to boost strong signal structures and to keep unmodified the other areas. For graph signals, a vertex located on an edge or a textured area has a high spectral distance with respect to its neighbors as compared to a vertex within a constant area. Therefore, we propose to construct a structure mask that accounts for the structures present in the graph signal. A normalized sum of distances within a local neighborhood is a good indicator of the graph signal structure, and is defined as $\delta\left(v_{i}\right)=\frac{\sum_{v_{j} \in B_{1}\left(v_{i}\right)} d_{E M D}\left(H\left(v_{j}\right), H\left(v_{i}\right)\right)}{\left|B_{1}\left(v_{i}\right)\right|}$ with $d_{E M D}$ the Earth Mover Distance between two signatures that are compact representations of local distributions [20]. To build $H\left(v_{i}\right)$, an histogram of size $N$ is constructed on the index graph signal $I$ as $H\left(v_{i}\right)=\left\{\left(w_{k}, m_{k}\right)\right\}_{k=1}^{N}$ within the set $B_{1}\left(v_{i}\right)$ where $m_{k}$ is the index of the $k$-th element and $w_{k}$ its appearance frequency. One has to note that $N \leq\left|B_{1}\left(v_{i}\right)\right|$ since identical values can be found within the set $B_{1}\left(v_{i}\right)$, and two signatures can have different sizes. To compute the EMD, ground distances are computed in the CIELAB color space. Finally, we define the structure mask of a graph signal as $M\left(v_{i}\right)=1+\frac{\delta\left(v_{i}\right)-\wedge \delta}{\vee \delta-\wedge \delta}$. One can notice that $M\left(v_{i}\right) \in[1,2]$ and will be close to 1 for constant areas and to 2 for ramp edges. Figure 3 presents examples of structure masks on two $3 \mathrm{D}$ colored graph signals. The structure mask is computed only once, and on the original graph signal $(I, \mathcal{P})=f$.

\section{EXPERIMENTAL RESULTS AND CONCLUSION}

We illustrate our approach on graph signals in the form 3D colored meshes that represent 3D scans of several person busts $^{1}$. Such scans have recently received much interest to generate $3 \mathrm{D}$ printed selfies and their perceived sharpness is

\footnotetext{
${ }^{1}$ Models from Cyberware and ReconstructMe.
} 

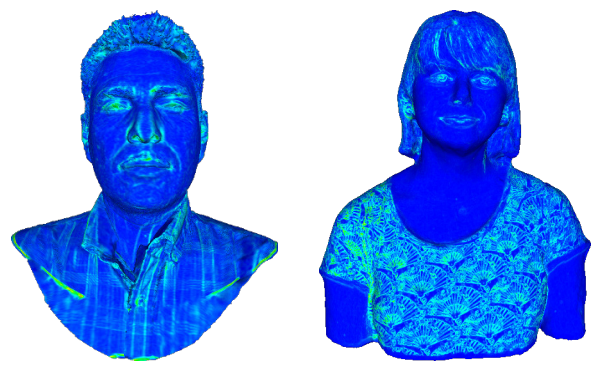

Fig. 3. Graph signal structure masks used to modulate the importance of detail enhancement. The original graph signals can be seen in the next figures.

of huge importance for final consumers. We have used $l=3$ levels of decomposition for computational efficiency. To assess objectively the benefit of our method, we measure the sharpness of the orignal signal $f$ and modified signal $\hat{f}$ with the TenenGrad criterion [21, 22], after adapting it to 3D colored meshes by using the morphological gradient (as in [22] for images): $T G(f)=\frac{1}{3|\mathcal{V}|} \sum_{v_{i} \in \mathcal{V}} \sum_{k=0}^{3}\left|\delta\left(f^{k}\right)\left(v_{i}\right)-\epsilon\left(f^{k}\right)\left(v_{i}\right)\right|$ where the morphological $\delta$ and $\epsilon$ are performed on each channel $f^{k}$ on a 1-hop. It has been shown in [22] that a higher value means a sharper signal and that this value is correlated with perceived sharpness. Figure 4 presents a first result with cropped and zoomed parts of the rendered mesh to better evaluate the enhancement. On all figures, please note that the enhancements are better visible at high resolution. It can

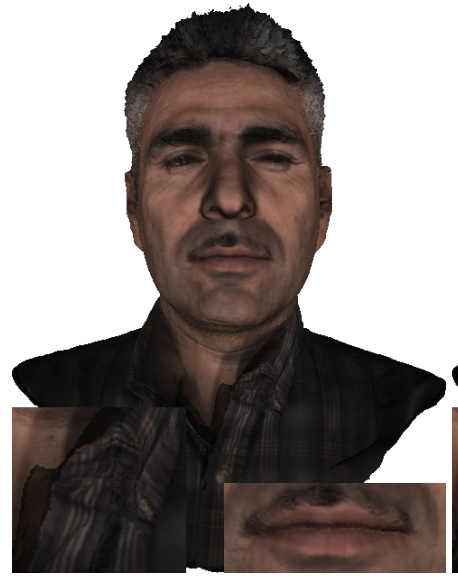

$T G(f)=3.86$
$T G(\hat{f})=5.40$

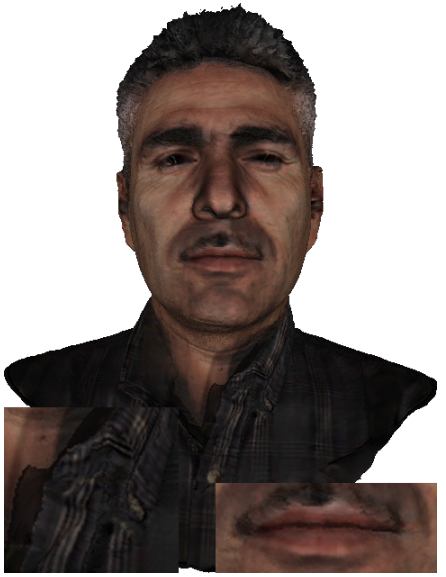

Fig. 4. Morphological colored mesh detail manipulation with cropped zoomed areas.

be seen that our approach has enhanced the local contrast without artifact magnification or detail loss. Figure 5 presents several other results. The results have objectively a much sharper appearance, and the enhancement is visually consistent. On the last mesh of Figure 5, the effect is all the more visible on the textured area of the shirt.
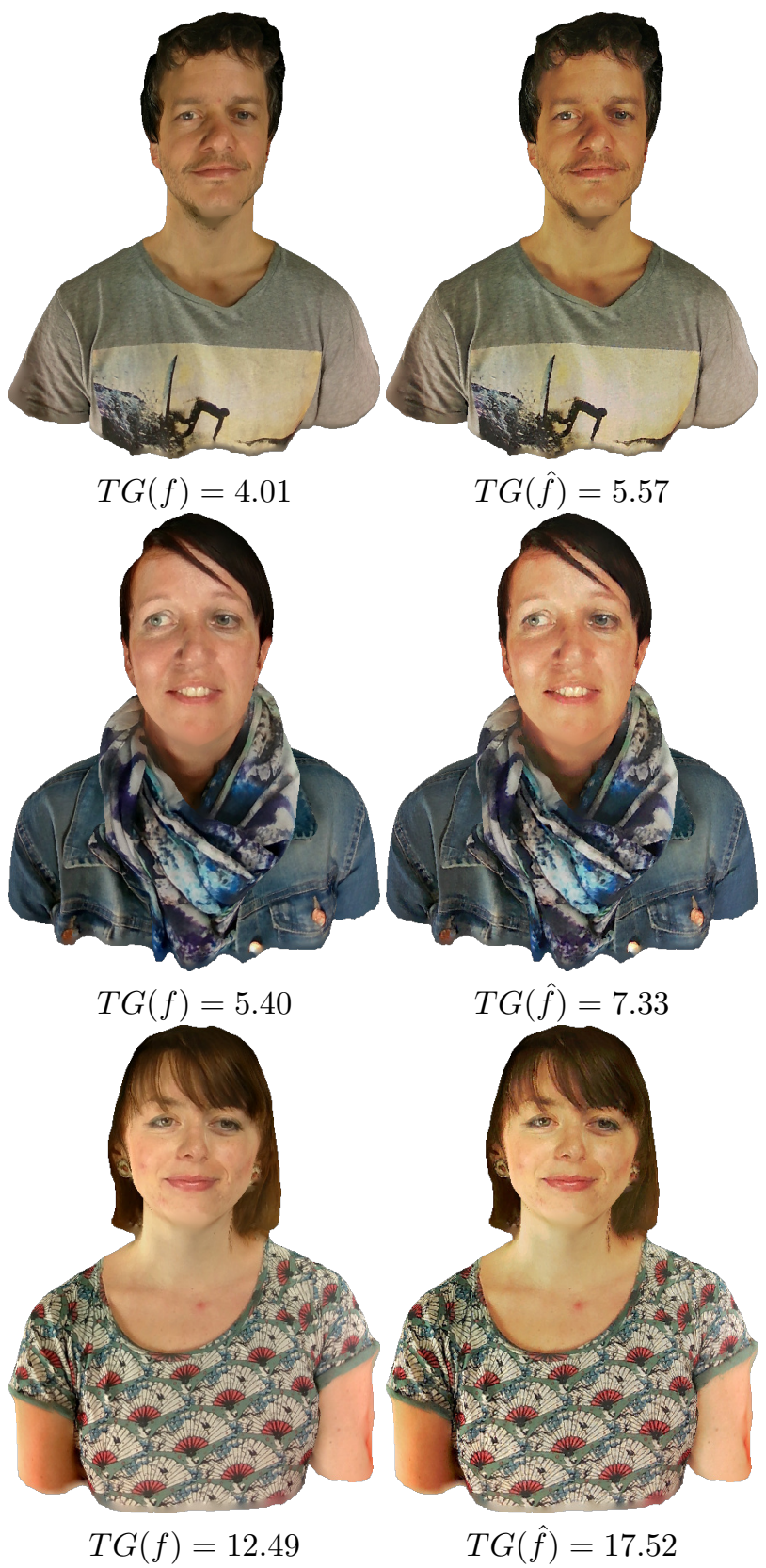

$T G(f)=12.49$

Fig. 5. Morphological colored mesh detail manipulation.

\section{CONCLUSION}

We have introduced an approach for 3D colored graph enhancement based on a morphological multi-layer decomposition of graph signals. The use of nonlinear detail manipulation with a structure mask enables to have an automatic method that produces visually appealing results of enhanced sharpness. 


\section{REFERENCES}

[1] Qi Zhang, Xiaoyong Shen, Li Xu, and Jiaya Jia, "Rolling guidance filter," in European Conference on Computer Vision ECCV, 2014, pp. 815-830.

[2] H. Cho, H. Lee, H. Kang, and S. Lee, "Bilateral texture filtering," ACM Transactions on Graphics, vol. 33, no. 4, pp. 128:1-128:8, 2014.

[3] E. S. L. Gastal and M. M. Oliveira, "Domain transform for edge-aware image and video processing," ACM Transactions on Graphics, vol. 30, no. 4, pp. 69, 2011.

[4] L. Xu, C. Lu, Y. Xu, and J. Jia, "Image smoothing via $L_{0}$ gradient minimization," ACM Transactions on Graphics, vol. 30, no. 6, pp. 174, 2011.

[5] S. Fleishman, I. Drori, and D. Cohen-Or, "Bilateral mesh denoising," ACM Transactions on Graphics, vol. 22, no. 3, pp. 950-953, 2003.

[6] Michael Kolomenkin, Ilan Shimshoni, and Ayellet Tal, "Prominent field for shape processing and analysis of archaeological artifacts," International Journal of Computer Vision, vol. 94, no. 1, pp. 89-100, 2011.

[7] Z. Afrose and Y. Shen, "Mesh color sharpening," Advances in Engineering Software, vol. 91, pp. 36 - 43, 2016.

[8] C. Ronse, "Why mathematical morphology needs complete lattices," Signal Processing, vol. 21, no. 2, pp. 129-154, 1990.

[9] J. Goutsias, H.J.A.M. Heijmans, and K. Sivakumar, "Morphological operators for image sequences," Computer Vision and Image Understanding, vol. 62, no. 3, pp. 326-346, 1995.

[10] O. Lézoray, "Complete lattice learning for multivariate mathematical morphology," Journal of Visual Communication and Image Representation, vol. 35, pp. 220235, 2016.

[11] A. Gersho and R.M. Gray, Vector Quantization and Signal Compression, Kluwer Academic, 1991.

[12] M. Belkin and P. Niyogi, "Laplacian eigenmaps for dimensionality reduction and data representation," Neural Computation, vol. 15, no. 6, pp. 1373-1396, 2003.

[13] A. Talwalkar, S. Kumar, M. Mohri, and H. A. Rowley, "Large-scale SVD and manifold learning," Journal of Machine Learning Research, vol. 14, no. 1, pp. 31293152, 2013.
[14] Z. Farbman, R. Fattal, D. Lischinski, and R. Szeliski, "Edge-preserving decompositions for multi-scale tone and detail manipulation," ACM Transactions on Graphics, vol. 27, no. 3, 2008.

[15] M. Hidane, O. Lézoray, and A. Elmoataz, "Graph signal decomposition for multi-scale detail manipulation," in International Conference on Image Processing (IEEE), 2014, pp. 2041-2045.

[16] R. A. Peters, "A new algorithm for image noise reduction using mathematical morphology," IEEE Transactions on Image Processing, vol. 4, no. 5, pp. 554-568, 1995.

[17] A. Choudhury and G. Medioni, "Hierarchy of nonlocal means for preferred automatic sharpness enhancement and tone mapping," Journal of the Optical Society of America A, vol. 30, no. 3, pp. 353-366, 2013.

[18] Sylvain Paris, Samuel W. Hasinoff, and Jan Kautz, "Local laplacian filters: edge-aware image processing with a laplacian pyramid," ACM Transactions on Graphics, vol. 30 , no. 4 , pp. $68,2011$.

[19] Hossein Talebi Esfandarani and Peyman Milanfar, "Fast multi-layer laplacian enhancement," CoRR, vol. abs/1606.07396, 2016.

[20] Yossi Rubner, Carlo Tomasi, and Leonidas J. Guibas, "The earth mover's distance as a metric for image retrieval," International Journal of Computer Vision, vol. 40, no. 2, pp. 99-121, 2000.

[21] X. Xu, Y. Wang, X. Zhang, S. Li, X. Liu, X. Wang, and J. Tang, "A comparison of contrast measurements in passive autofocus systems for low contrast images," Multimedia Tools and Applications, vol. 69, no. 1, pp. 139-156, 2014.

[22] A. Choudhury and G. Medioni, "Perceptually motivated automatic sharpness enhancement using hierarchy of non-local means," in IEEE International Conference on Computer Vision Workshops, ICCV, 2011, pp. 730737. 